\title{
Sincronización de sistemas activos en procesos de clasificación de objetos por color
}

\section{Synchronization of active systems in object classification process by color}

\author{
RODRÍGUEZ-FRANCO, Martín Eduardo†*, MARTÍNEZ-ROMERO, Adrian, NÁJERA-MONTOYA, \\ Adolfo Ángel y LÓPEZ-ÁLVAREZ, Yadira Fabiola
}

Universidad Tecnológica del Norte de Aguascalientes. Av. Universidad \#1001, Estación Rincón, 20400, Rincón de Romos, Ags

ID $1^{\text {er }}$ Autor: Martín Eduardo, Rodríguez-Franco / ORC ID: 0000-0002-6804-4777, Researcher ID Thomson: T-15392018, CVU CONACYT ID: 660892

ID $1^{\text {er }}$ Coautor: Adrian, Martínez-Romero / ORC ID: 0000-0002-1310-1287, Open ID: 114501414588597884047, CVU CONACYT ID: 1005146

ID $2^{\text {do }}$ Coautor: Adolfo Ángel, Nájera-Montoya / ORC ID: 0000-0002-2076-8039, Open ID: 111662003104936007784, CVU CONACYT ID: 1005155

ID $3^{\text {er }}$ Coautor: Yadira Fabiola, López-Álvarez / ORC ID: 0000-0002-9041-1908, Researcher ID Thomson: T-1555-2018, CVU CONACYT ID: 375952

DOI: $10.35429 /$ JTIP.2020.11.4.21.31

Recibido 30 de Septiembre, 2020; Aceptado 20 Diciembre, 2020

Resumen

El presente documento propone una aplicación de la teoría de sistemas de control en eventos discretos, y su sintetización empleando la especificación GRAFCET, para la gestión de un prototipo de sistema de manejo y clasificación de objetos por color. Se expone la interacción y la sincronización entre un robot en configuración angular y una banda transportadora, como elementos activos, con la finalidad de establecer una secuencia de tareas a ejecutar; la cual principia con el ingreso de un objeto al proceso, para finalizar con el posicionamiento del mismo en el depósito respectivo, una vez identificado su color. Se muestra el desarrollo del prototipo para pruebas, la definición y la programación del sistema de control del proceso a partir de la metodología sugerida y la integración de la interfaz gráfica que permita la visualización de su estado hacia el usuario. Los resultados sugieren que la aplicación de métodos formales, como GRAFCET, promueven la conformación de un sistema de control funcional, estructurado, escalable, distribuido y concurrente, al permitir la adecuada descentralización de las tareas que cada elemento activo debe ejecutar y a la vez determine su oportuna acción, sin interferencias entre sí.

Sincronización, Clasificación por color, Gráfico
funcional de control de etapas/transiciones
(GRAFCET)

\begin{abstract}
This document proposes an application of the theory of control systems in discrete events, and its synthesis using GRAFCET specification, for the management of a prototype system for handling and classifying objects by color. The interaction and synchronization between a robot in angular configuration and a conveyor belt are exposed as active elements, in order to establish a sequence of tasks to be executed; which begins with the entry of an object to the process, to end with the positioning of the same in the respective deposit, once its color has been identified. The development of the prototype for testing, the definition and programming of the process control system based on the suggested methodology and the integration of the graphical interface that allows the user to view its status is shown. The results suggest that the application of formal methods, such as GRAFCET, promote the establishment of a functional, structured, scalable, distributed and concurrent control system, by allowing adequate decentralization of the tasks that each active element must execute and at the same time determine its timely action, no interference with each other.
\end{abstract}

Synchronization, Color classification, Graphe fonctionnel de commande des étapes et transitions (GRAFCET)

Citación: RODRÍGUEZ-FRANCO, Martín Eduardo, MARTÍNEZ-ROMERO, Adrian, NÁJERA-MONTOYA, Adolfo Ángel y LÓPEZ-ÁLVAREZ, Yadira Fabiola. Sincronización de sistemas activos en procesos de clasificación de objetos por color. Revista de Tecnologías en Procesos Industriales. 2020. 4-11: 21-31

\footnotetext{
* Correspondencia del Autor (martin.rodriguez@utna.edu.mx)

$\dagger$ Investigador contribuyendo como primer autor.
} 


\section{Introducción}

Un sistema activo se presenta como una serie o colección de elementos cuya característica primordial es la ejecución de alguna tarea de procesamiento o movimiento de objetos, dentro de una operación en la cual sea insertado (Huang, Chiba, Arai, Ueyama, \& Ota, 2015). A nivel industrial, el desempeño de actividades tales como: posicionamiento, sujeción, transportación y distribución, o en general, manejo de materiales, requieren la utilización de un sistema activo para cumplir con los objetivos logísticos internos previstos (Hrúz \& Mrafko, 2003) (Fitouhi, Nourelfath, \& Gershwin, 2017). Es así, que un sistema activo necesita combinar su acción particular con la de otros elementos, para definir una secuencia de procesamiento y transferencia de materias primas hacia operaciones de transformación, de materiales en transición a otros procesos que les otorguen mayor valor, o en su defecto, de productos terminados hacia almacenes, para la continuación de la cadena de suministro (Campos, Santos, de Souza, \& da Silva, 2013).

Para obtener por beneficio la optimización de recursos humanos, materiales y tecnológicos, así como del tiempo requerido en la ejecución de un proceso específico, es necesario realizar una adecuada planificación de la sincronización de los sistemas activos considerados (Silva, Ribeiro, \& Teixeira, 2017) (Ore, Hansson, \& Magnus, 2017). Tal efecto, habrá de contemplar características de concurrencia que permitan suponer la acción de un sistema activo específico mientras otro distinto también esté en funcionamiento, sin que sus operaciones lleguen a interferir entre sí (Björnsson, Jonsson, \& Johansen, 2018). Para lo cual, se vuele inminente contar con un sistema de percepción que brinde las señales de retroalimentación del estado del proceso propias, para la adecuada gestión de las actividades del sistema total que les integre (Pichard, Ben Rabah, Carre-Menetrier, \& Riera, 2016).

El uso de una metodología formal como GRAFCET promueve, a partir de conjuntar la información proveniente de sensores, la generación de las señales de consigna que lleven a los sistemas activos empleados a adoptar los comportamientos requeridos, en cumplimiento de una tarea de proceso previamente establecida (Alvarez, Burgos, Sarachaga, Estévez, \& Marcos, 2012).
Por su parte, la aplicación de sinópticos de control, provista por esta técnica, en combinación con la representación gráfica de las relaciones entre señales de entrada y salida, establecen una clara definición y aseguramiento de la secuencia que se desee adoptar. Tal operación progresiva puede ser directamente interpretable para su programación en los controladores de proceso utilizados(Qamsane, El Hamlaoui, Tajer, \& Philippot, 2017); otorgando adicionalmente, la capacidad de descentralizar su función mediante la disposición de múltiples unidades de control, coordinadas dentro de una red industrial distribuida (Endsley, Almeida, \& Tilbury, 2006) (Schumacher, Schröck, \& Fay, 2013).

La metodología GRAFCET es empleada hoy en día como una alternativa fehaciente para aplicaciones que requieren asegurar, no solamente una sincronización básica entre unos pocos sistemas activos, como es el caso del trabajo expuesto en el presente documento y que a continuación se detalla; sino donde interviene un gran número de éstos, aumentando la complejidad del sistema total y no por ello, de su control (Riera, Coupat, Philippot, Annebique, \& Gellot, 2014) (Qamsane, Tajer, \& Philippot, 2016) (Schneider, Peßlera, \& Terkaj, 2019).

\section{Antecedentes}

Desde tiempo atrás, la cooperación entre profesores y alumnos de la Universidad Tecnológica de Norte de Aguascalientes ha permitido la planeación, el desarrollo y la puesta en operación de implementos físicos que incentivan la aplicación práctica de conocimientos teóricos adquiridos en el aula. Tal orientación de la educación tecnológica, basada en proyectos, ha llevado a encarar al estudiante participante con la necesidad de poner en práctica gran parte del conocimiento adquirido durante su formación profesional como Técnico Superior Universitario en la creación y gestión de prototipos tecnológicos que apoyen al desarrollo de las competencias propias.

Una motivación complementaria a este tipo aprendizaje aplicado, es la orientación del proceder del estudiante hacia la adopción y aplicación de metodologías formales, mismas que promuevan un sustento para la resolución de problemáticas que puedan hacerles frente una vez insertados en el campo industrial. 
De forma adicional, la conformación de prototipos tecnológicos ha determinado ser una opción válida en situaciones donde la carencia de equipamiento industrial de las instituciones de educación superior, no debiera ser considerada una limitante para dejar de brindar el conocimiento que permita al estudiante el desarrollo de las competencias profesionales que el programa educativo cursado ampare.

Es así, que el presente trabajo representa la continuidad dada al desarrollo de un prototipo de sistema de manejo y clasificación de materiales, previamente conformado, al dotarlo ahora de la capacidad de discernir entre distintas clases de color presentes en un objeto susceptible de ser clasificado. La adición de esta funcionalidad preserva el propósito de brindar un apoyo al docente en el proceso de enseñanzaaprendizaje de asignaturas técnicas tales como Robótica y Manufactura Flexible valoradas como parte del programa educativo de TSU en Mecatrónica área Sistemas de Manufactura Flexible.

\section{Distribución del prototipo}

Se dispone de un prototipo físico de manejo y clasificación de materiales por tamaño, el cual fuera adaptado para el desempeño de la tarea de clasificación de objetos, ahora bajo el análisis de la característica de color. Los elementos activos empleados son un brazo robótico de tres grados de libertad, en configuración angular, y una banda transportadora, en cuyos extremos fueron fijados un par de sensores de tipo infrarrojo. La localización de ambos puntos extremos de la banda transportadora dentro del área de trabajo del robot permitirá a su efector final, en este caso un gripper, alcanzar tales posiciones según sea requerido por la secuencia de proceso a ejecutar. Además, de considerar dos áreas adicionales en el propio prototipo, una para el abastecimiento de material hacia el proceso y otra para el almacenamiento de éste, de forma posterior a determinar su pertenencia a una clase en particular, dada la característica de estudio.

Cabe mencionar, la necesidad de definir las ubicaciones específicas que cada sistema activo posee con respecto a otros en el prototipo; esto con el objetivo de establecer las posiciones que habrán de ser alcanzadas por el robot empleado durante el desempeño de su función.
Tal situación, elimina la posibilidad de que las posiciones propuestas para el gripper lleguen a variar durante la ejecución del proceso, originando colisiones. Para lograr lo anterior, se realizó el montaje de los sistemas activos en una placa sólida que permitiera además, la adecuada delimitación de las áreas de abastecimiento y almacenamiento de material, tal como puede observarse en el esquema de la figura 1. Es necesario resaltar, que el ingreso de material hacia el área de abastecimiento se realiza de forma manual.

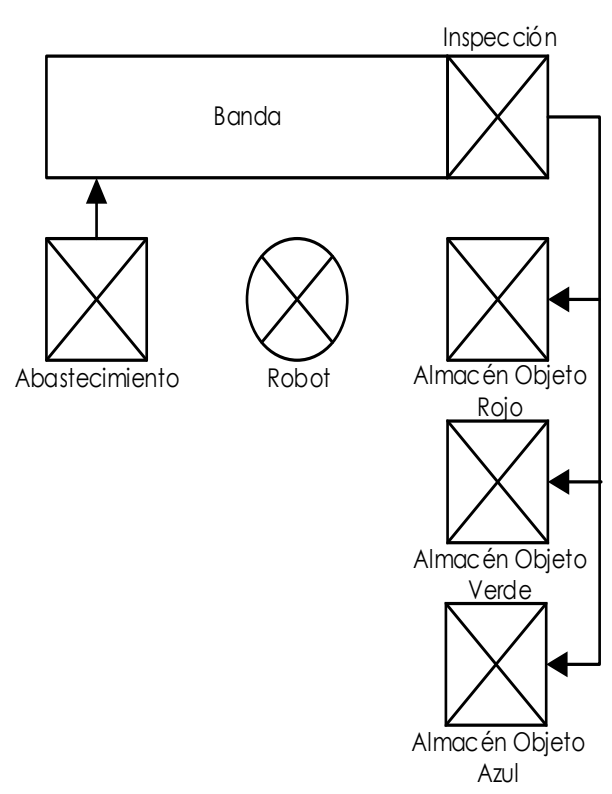

Figura 1 Distribución del prototipo

Fuente: Elaboración Propia, 2020

\section{Parámetros y variables de proceso}

Los parámetros del proceso proceden del botón dispuesto para la habilitación general del sistema, así como de sensores infrarrojos que fueron colocados en posiciones específicas del prototipo; a fin de brindar información sobre la presencia de objetos en éstas, al ver interrumpido el haz de luz transferido entre ambos elementos del sensor. Otras señales empleadas corresponden al alcance de ciertas posiciones funcionales por parte del efector final del robot, el estado del gripper utilizado o la finalización de tareas dadas (interbloqueos). Finalmente, se resalta la acción del sensor de color, el cual emite señales eléctricas relacionadas con la frecuencia electromagnética respectiva al color detectado en la superficie de cada objeto analizado, y que detona la decisión del destino posterior del mismo. Los parámetros del proceso, señales e identificadores se presentan en la tabla 1 . 


\begin{tabular}{|l|l|l|}
\hline \multicolumn{1}{|c|}{ Identificador } & \multicolumn{1}{|c|}{ Señal } & \multicolumn{1}{|c|}{ Sistema } \\
\hline PB0 & Encendido del sistema. & Sistema general \\
\hline ZS0 & Sensor de abastecimiento. & Sistema general \\
\hline ZS1 & Sensor de extremo 1. & $\begin{array}{l}\text { Banda } \\
\text { transportadora }\end{array}$ \\
\hline ZS2 & Sensor de extremo 2. & $\begin{array}{l}\text { Banda } \\
\text { transportadora }\end{array}$ \\
\hline X0dn & En posición de reposo. & Robot \\
\hline X1dn & $\begin{array}{l}\text { En posición de extremo 1 } \\
\text { en la banda. }\end{array}$ & Robot \\
\hline X2dn & $\begin{array}{l}\text { En posición de extremo 2 } \\
\text { en la banda. }\end{array}$ & Robot \\
\hline X3dn & $\begin{array}{l}\text { En de } \\
\text { almacenamiento clase 1 } \\
\text { Rojo). }\end{array}$ & Robot \\
\hline X4dn & $\begin{array}{l}\text { En posición de } \\
\text { almacenamiento clase 2 } \\
\text { (Verde). }\end{array}$ & Robot \\
\hline X5dn & $\begin{array}{l}\text { En posición de } \\
\text { almacenamiento clase 3 } \\
\text { (Azul). }\end{array}$ & Robot \\
\hline Gcldn & Gripper cerrado. & Gripper \\
\hline Gopdn & Gripper abierto. & Gripper \\
\hline C1 & Identificación de clase 1. & Inspección \\
\hline C2 & Identificación de clase 2. & Inspección \\
\hline C3 & Identificación de clase 3. & Inspección \\
\hline TM0dn & $\begin{array}{l}\text { Tiempo para identificación } \\
\text { concluido. }\end{array}$ & Inspección \\
\hline
\end{tabular}

Tabla 1 Parámetros del proceso Fuente: Elaboración Propia, 2020

Las variables son determinadas por el controlador de cada sistema activo para la adopción, por parte de éste, de un comportamiento particular atendiendo a la tarea que se requiera desempeñar. Lo que refiere, en el caso del robot, a una localización definida de su efector final para realizar acciones de sujeción o liberación de objetos, respectivamente; o tratándose de la banda transportadora, su activación o desactivación, para la conducción de un objeto entre sus dos extremos. En la tabla 2 se observan las variables de proceso, sus respectivas señales e identificadores.

\begin{tabular}{|l|l|l|}
\hline Identificador & \multicolumn{1}{|c|}{ Señal } & \multicolumn{1}{c|}{ Sistema } \\
\hline X0 & A posición de reposo. & Robot \\
\hline X1 & A posición de abastecimiento. & Robot \\
\hline X2 & $\begin{array}{l}\text { A posición de extremo 1 de la } \\
\text { banda. }\end{array}$ & Robot \\
\hline X3 & $\begin{array}{l}\text { A posición de extremo 2 de la } \\
\text { banda. }\end{array}$ & Robot \\
\hline X4 & $\begin{array}{l}\text { A posición de almacenamiento } \\
\text { clase 1 (Rojo). }\end{array}$ & Robot \\
\hline X5 & $\begin{array}{l}\text { A posición de almacenamiento } \\
\text { clase 2 (Verde). }\end{array}$ & Robot \\
\hline X6 & $\begin{array}{l}\text { A posición de almacenamiento } \\
\text { clase 3 (Azul). }\end{array}$ & Robot \\
\hline X7 & A posición de reposo. & Robot \\
\hline X8 & A posición de reposo. & Robot \\
\hline X9 & A posición de reposo. & Robot \\
\hline Gcl & Cerrar gripper. & Gripper \\
\hline Gop & Abrir gripper. & Gripper \\
\hline MT & Activar motor. & $\begin{array}{l}\text { Banda } \\
\text { transportadora }\end{array}$ \\
\hline TM0 & Activar temporizador. & Sistema general \\
\hline
\end{tabular}

Tabla 2 Variables del proceso

Fuente: Elaboración Propia, 2020

\section{Operación del sensor de color}

Desde un punto de vista físico, el color de un objeto es producto de la capacidad del material del cual está hecho, para absorber la luz. Esta propiedad corresponde a la composición de los colores respectivos a las ondas reflejadas en dicho color. Tal composición se refiere entonces, a las combinaciones de los colores base: rojo, verde y azul. En este caso, el sensor de color utilizado (figura 2) es del tipo basado en filtros, mismo que proporciona una salida de voltaje directamente proporcional a la irradiación, es decir, a las longitudes de onda detectadas por un grupo de tres fotodiodos, cada uno con un filtro de color, para los tres tipos mencionados.

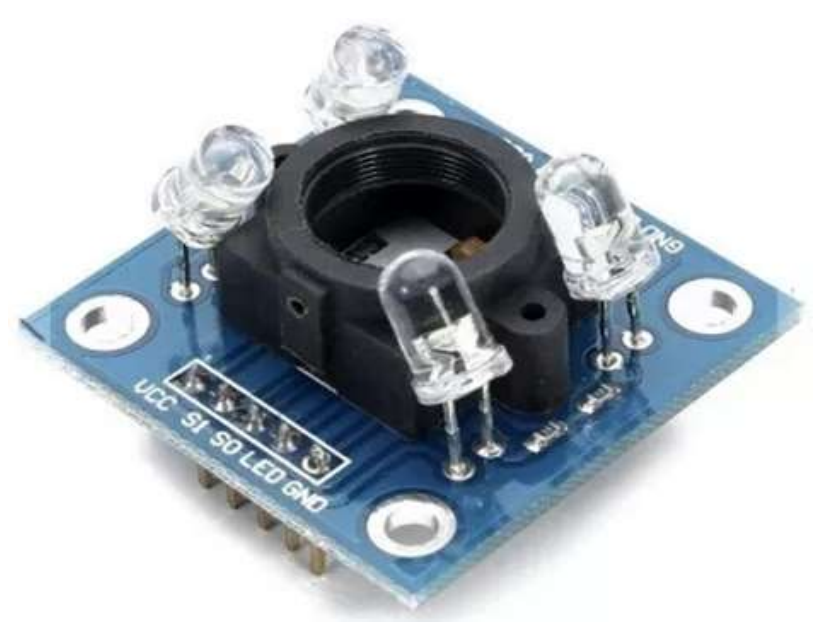

Figura 2 Sensor de color RGB TCS3200

Fuente (Corona Ramírez, Abarca Jiménez, \& Mares Carreño, 2014)

El tipo de sensor utilizado posee una fuente de luz blanca cuyo objetivo es apoyar en la claridad del color analizado, procurando no incrementar de forma indirecta la magnitud de alguna de sus componentes base. Atendiendo a la magnitud de cada componente de color detectada en la superficie del objeto, se emite una señal de voltaje de salida proporcional, a través de la terminal correspondiente. Se dispone de las terminales $\mathrm{V}_{\mathrm{R}}, \mathrm{V}_{\mathrm{G}} \mathrm{y} \mathrm{V}_{\mathrm{B}}$, respectivamente. Tales señales de voltaje son leídas en forma binaria, a 8 bits, y las múltiples combinaciones de éstas corresponden a los diferentes colores que el sensor puede medir. La componente de color predominante será aquella en la que se genere una mayor magnitud de voltaje de salida (Corona Ramírez, Abarca Jiménez, \& Mares Carreño, 2014). 


\section{Secuencia del proceso}

El proceso propuesto principia con el robot empleado en posición de reposo y con el gripper abierto. Su función dará comienzo una vez que sea activado el botón de arranque respectivo, además de ser detectada una pieza en el área de abastecimiento del prototipo. Esto es posible a partir de la interrupción de la señal existente entre un par de sensores infrarrojos ahí dispuestos para tal función. La presencia de la pieza incidirá el movimiento del robot angular, para recuperar y guiar a la misma hacia un primer extremo de la banda transportadora, donde la liberará.

El uso de otro par de sensores infrarrojos, activados al detectar pieza en el primer extremo de la banda, determinará el movimiento del robot para destinarlo a su posición de reposo nuevamente. Al arribar el robot a esta última posición, se activará el giro en la banda transportadora, para desplazar la pieza al extremo contrario de donde fuera inicialmente colocada. Cabe mencionar, que el robot permanecerá en su posición de reposo mientras la pieza recorre la extensión de la banda.

Una vez alcanzada la última posición propuesta por la pieza, la banda detendrá su recorrido, manteniéndola en dicha localización durante un pequeño lapso de tiempo. Otro par de sensores infrarrojos hace posible la detección del arribo de pieza al segundo extremo de la banda transportadora. El sensor de color permitirá la identificación de tal característica en la superficie de cada pieza que hayan sido transportada hasta tal extremo de la banda, distinguiendo entre tres clases distintas: roja, verde y azul.

Transcurrido el tiempo de detección de la clase a la que pertenece la pieza evaluada, el robot se desplazará desde su posición de reposo prescribiendo una trayectoria que permita recuperar nuevamente a la pieza en cuestión, y destinarla hacia el depósito correspondiente. Por lo cual, se contempla la disposición de tres depósitos, uno respectivo a cada clase establecida, y por ende, la propuesta de una trayectoria distinta en cada caso, ejecutada por el robot, que lleve a la pieza a cada uno de estos, según sea el resultado del análisis. Concluida la tarea de clasificación, el robot retomará su posición de reposo, a la espera del ingreso de una nueva pieza al proceso.
Posterior a la descripción textual de la secuencia del proceso propuesto, será necesaria la representación de las señales de entrada y salida que intervienen en el mismo. Éstas permitirán el ingreso de información al controlador, para su procesamiento y la generación de las señales que propicien la activación de las cargas eléctricas que constituyen a cada uno de los sistemas activos; en este caso específico, los motores eléctricos de corriente directa y servomotores utilizados.

\section{Representación del controlador}

La teoría de sistemas de control en eventos discretos se apoya en la especificación GRAFCET (gráfico funcional de control de etapas y transiciones) para describir el comportamiento de una secuencia de proceso, en la cual pueden intervenir múltiples sistemas activos (Zaytoon, 2010). Esto es posible, a través de la definición y la representación de las acciones realizadas por los actuadores o elementos finales, en relación con condiciones asociadas a señales provenientes de sensores o a la conclusión de tareas específicas, según corresponda (Zhang, Yang, Zhang, \& Shi, 2012).

Un gráfico básico GRAFCET consta de dos elementos principales: etapas, representadas por rectángulos, y transiciones, mostradas como líneas perpendiculares a la unión entre dos etapas consecutivas. La combinación de ambos elementos descritos determina el flujo del proceso de principio a final (Alvarez, Burgos, Sarachaga, Estévez, \& Marcos, 2012). Cada etapa podrá poseer un estado activo o, en su defecto, inactivo; según respecte a la secuencia dada. El concepto de etapa inicial se adopta para representar a los elementos que habrán de estar activos al comienzo de la ejecución del proceso propuesto. Siempre que exista una condición habilitada que se encuentre asociada a una transición, esta permitirá la operación de receptividad, lo que provocará que la etapa o etapas posteriores sean activadas (Jamhour \& Garcia, 2012) (Schumacher, Schröck, \& Fay, 2013).

En el presente caso, una vez definidos los parámetros, las variables y la secuencia del proceso, se realiza la representación del esquema GRAFCET correspondiente, según se muestra en la figura 3. 
Tal modelo gráfico puede ser adecuadamente interpretado y transformado, por medio de un lenguaje de programación de conveniencia, para su implantación dentro del controlador de proceso; con lo cual se asegure la gestión física de los sistemas activos integrantes (Qamsane, El Hamlaoui, Tajer, \& Philippot, 2017).

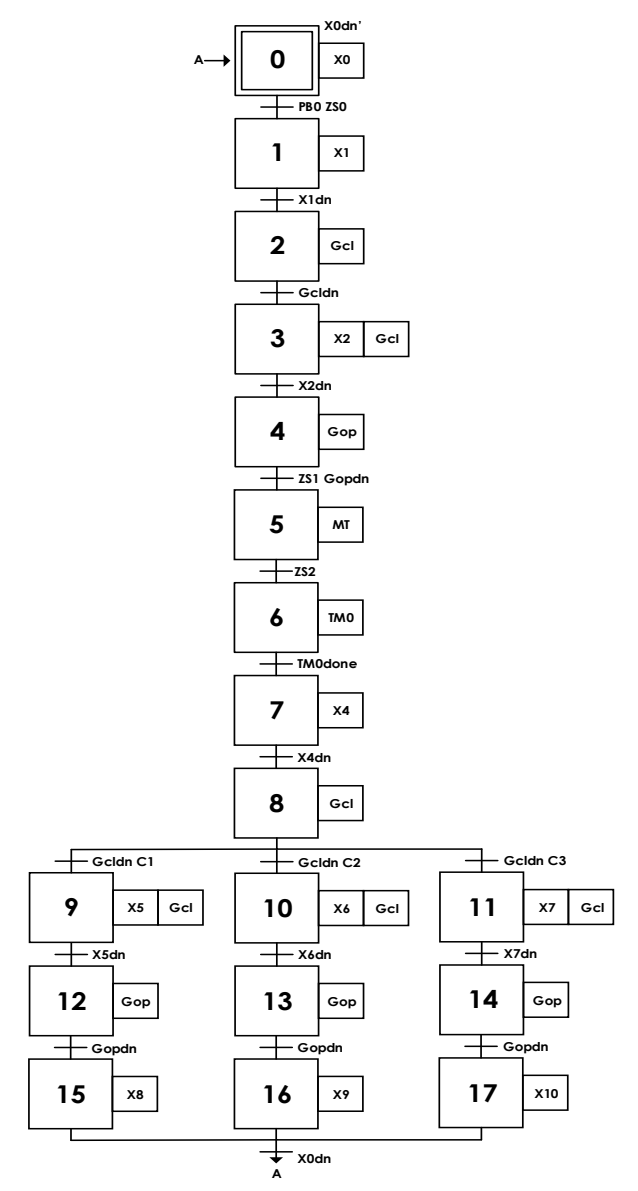

Figura 3 GRAFCET del proceso de clasificación de objetos por color

Fuente: Elaboración Propia, 2020

Un aspecto resaltable del empleo de esta metodología, es el hecho de representar de forma intuitiva, simple y clara, características estructurales de la secuencia de un proceso bajo estudio, tales como selección y simultaneidad. Y, ha sido tanta la importancia de GRAFCET como medio para la automatización de sistemas, que se encuentra regulado bajo el estándar IEC 60848 (Riera, Annebicque, Gellot, Philippot, \& Benlorhfar, 2012) (Schumacher, Schröck, \& Fay, 2013).

\section{Programación del controlador}

El controlador del proceso se encuentra integrado por dos tarjetas electrónicas Arduino UNO en comunicación con la interfaz gráfica, desarrollada para la gestión del proceso.
La primera tarjeta recolecta las señales emitidas por los sensores dispuestos en el prototipo, además de gestionar la activación de la banda transportadora. La segunda tarjeta envía las consignas hacia los actuadores que comandan las articulaciones y el gripper del robot, asegurando el desempeño de su tarea. Ambas tarjetas fungen como puertos de entrada y salida para el sistema de control implementado. La arquitectura del controlador de proceso se aprecia en la figura 4.

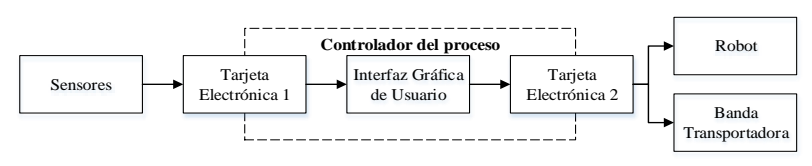

Figura 4 Arquitectura del controlador Fuente: Elaboración Propia, 2020

Para esta aplicación de clasificación, es claro percibir que la primera tarjeta será la encargada de interpretar el color determinado en la pieza analizada, a su arribo al área de inspección; esto a partir de la lectura de las señales emitidas por las terminales de salida $\left(V_{R}\right.$, $\mathrm{V}_{\mathrm{G}}, \mathrm{V}_{\mathrm{B}}$ ) del sensor dispuesto para tal fin. Posterior a la plena identificación de la clase respectiva, la segunda tarjeta comandará al robot para cumplir con la secuencia específica que le lleve a depositar al objeto en cuestión en el depósito correspondiente.

La comunicación entre ambas tarjetas y una computadora se realiza a través de puertos USB mediante el dispositivo UART (Universal Asynchronous Receiver-Transmitter) adaptado a las tarjetas Arduino empleadas. Por tanto, será la tarea principal de la computadora disponer de los recursos adecuados para la ejecución de la interfaz gráfica que gestione el proceso, a partir del monitoreo y control de las señales implicadas en el mismo.

\section{Interfaz gráfica para gestión}

Un instrumento virtual desarrollado en el software LabVIEW es la base de la interfaz gráfica para la gestión de las señales que interactúan en el prototipo constituido. Fue necesario adaptar los sinópticos adecuados que permitieran al sistema de gestión ser intuitivo, accesible y amigable con el usuario. Además, de asegurar las funciones de retroalimentación de la condición del proceso en tiempo real y de ejecución automática y oportuna de las tareas que habrán de desempeñarse para cumplir con la secuencia establecida.

RODRÍGUEZ-FRANCO, Martín Eduardo, MARTÍNEZ-ROMERO, Adrian, NÁJERA-MONTOYA, Adolfo Ángel y LÓPEZ-ÁLVAREZ, Yadira Fabiola. Sincronización de sistemas activos en procesos de clasificación de objetos por color. Revista de Tecnologías en Procesos Industriales. 2020 
La figura 5 expone la interfaz desarrollada, delimitando las secciones que agrupan a los elementos de señalización incorporados, cuya función se detallan a continuación.

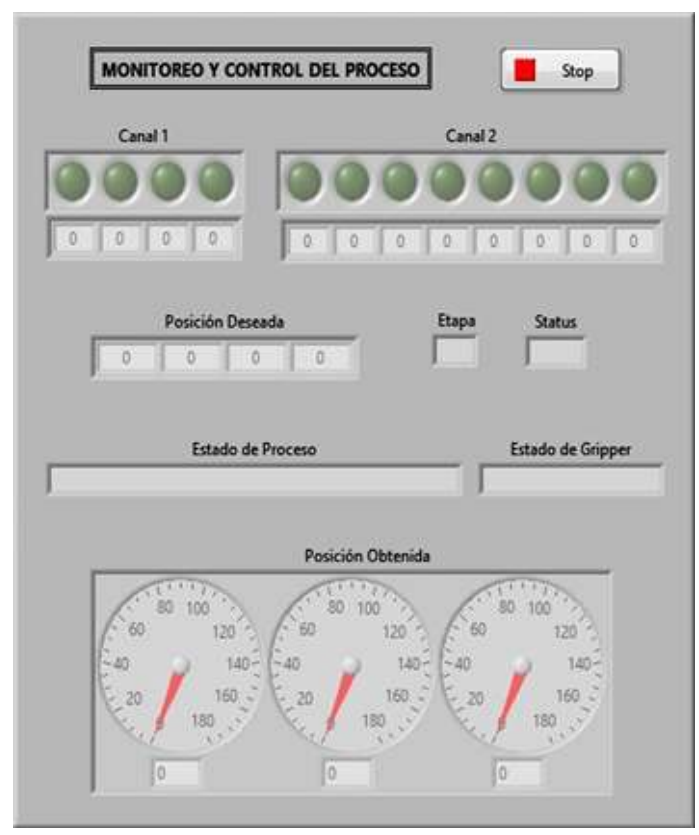

Figura 5 Interfaz gráfica para gestión del proceso Fuente: Elaboración Propia, 2020

El canal 1 expone la información que el controlador del proceso emitirá como señales de salida para la habilitación de los sistemas activos, asociadas al cumplimiento de las funciones del robot. Mientras el canal 2 representa la condición de los parámetros del proceso, gestionados desde la tarjeta 1, promoviendo el desempeño de alguna función particular por parte del sistema. Posición deseada, muestra la información de posición angular que el controlador envía a cada articulación que conforma al robot, y que le otorga movilidad.

Por su parte, etapa y estatus, permiten al usuario identificar, en forma codificada, que tarea se encuentra en ejecución, así como visualizar del estado particular del gripper empleado. Estado del proceso y del gripper emiten un mensaje de texto que alerta al usuario acerca del estado actual de la secuencia del proceso, indicando tanto la localización del efector final del robot en las secciones del prototipo, así como el estado tanto de la banda como del gripper. Finalmente, posición obtenida retroalimenta el valor real de las variables articulares del robot, indicando la evolución que cada una va teniendo conforme el controlador ordena la modificación en la posición cartesiana del gripper del robot.
Cabe mencionar, que cada una de las posiciones que habrá de adquirir el gripper del robot durante su evolución ha sido previamente propuesta, procurando en todo momento limitar la ocurrencia de colisiones con el propio robot o la banda transportadora. Para lo cual, fue contemplada una ubicación relativa adecuada del robot, y en específico de su gripper, con respecto a la banda transportadora y los sensores dispuestos; a manera de propiciar un daño mínimo en la instalación por describir algún movimiento erróneo, durante la ejecución de las pruebas.

\section{Resultados de operación del prototipo físico}

Una vez en operación, el comportamiento de la interfaz gráfica desarrollada fue el adecuado, cumpliendo su función al retroalimentar al usuario acerca de la condición del proceso desempeñado por el prototipo, durante la ejecución de la secuencia propuesta. Tal funcionamiento se constata en las figuras $6,7,8$ y 9 , cuya evidencia corrobora la realización de la acción asignada a cada elemento constitutivo del sistema físico implementado, de acuerdo al estado que el mismo debida adquirir; lo cual transcurrió sin errores.

Se garantizó la oportuna continuidad de las etapas definidas a partir del esquema GRAFCET, propuesto para el control del proceso implementado (figura 3); una vez habilitadas las transiciones requeridas para cada caso específico. De esta manera, se constata que la organización proporcionada por la especificación empleada garantiza el adecuado seguimiento en la sucesión de operaciones planeadas, y representadas en el mismo diagrama, según sea indicado.

En la figura 6 se presenta el posicionamiento del robot en una localización segura o de reposo, como el primer comportamiento que el sistema alcanza posterior a su energización. Asimismo, se aprecia que el gripper permanece abierto durante esta etapa, con la finalidad para dar cumplimiento a su tarea posterior. La siguiente evolución en el movimiento del robot le lleva hacia el área de abastecimiento del sistema, al ser detectado un objeto en la misma, para realizar su recuperación, según se muestra en la figura 7 . 


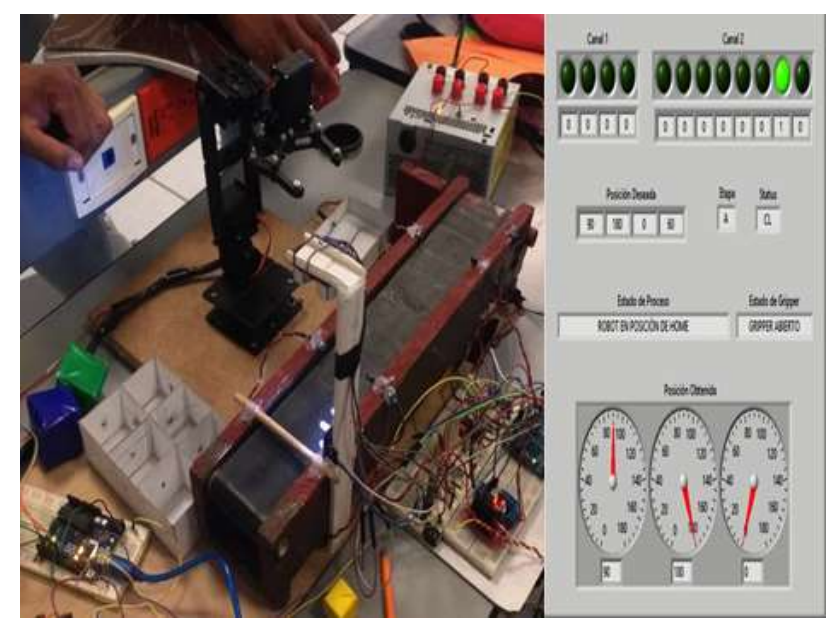

Figura 6 Robot en reposo

Fuente: Elaboración Propia, 2020

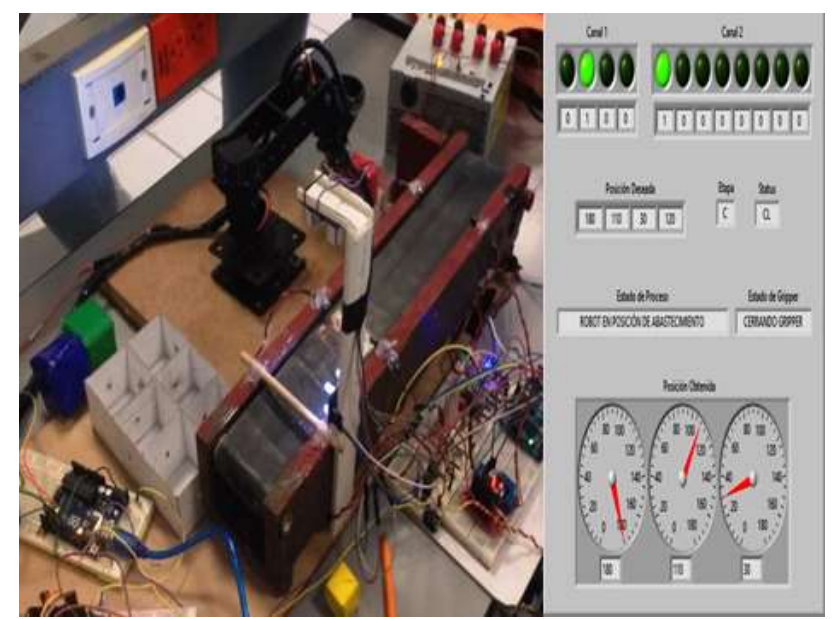

Figura 7 Recuperación de pieza de abastecimiento Fuente: Elaboración Propia, 2020

La figura 8 expone la segunda evolución en el movimiento del robot, misma que destina el objeto detectado hacia el primer extremo de la banda, para liberarla sobre este punto. De esta manera, la banda transportadora se activa para transferir el objeto hacia el extremo contrario, donde se localiza el área de inspección. Después de realizar el análisis de color respectivo, y una vez identificada la clase a la que pertenece el objeto en estudio, se guía al robot para sujetarlo en esta última posición, según se visualiza en la figura 9, para destinarlo hacia el depósito que le corresponda.

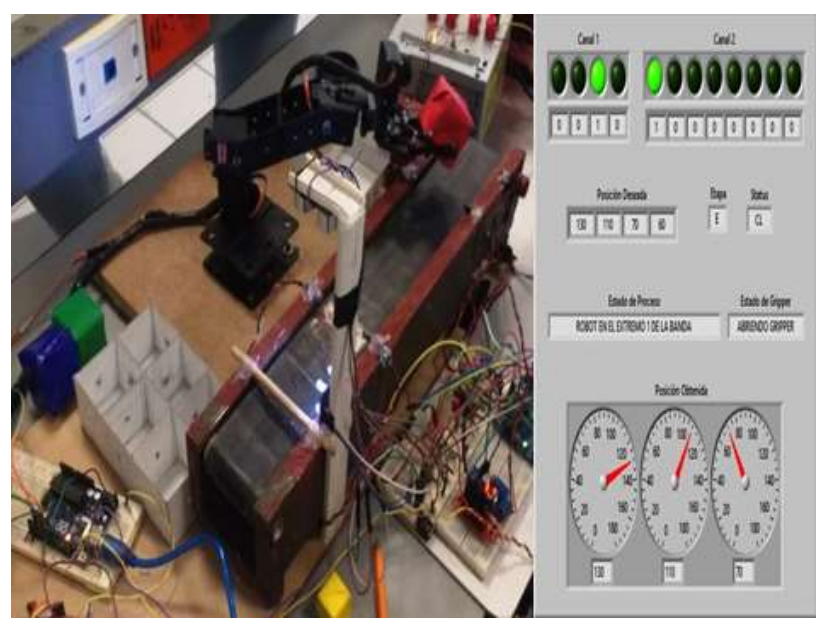

Figura 8 Posicionamiento del robot sobre el primer extremo de la banda

Fuente: Elaboración Propia, 2020

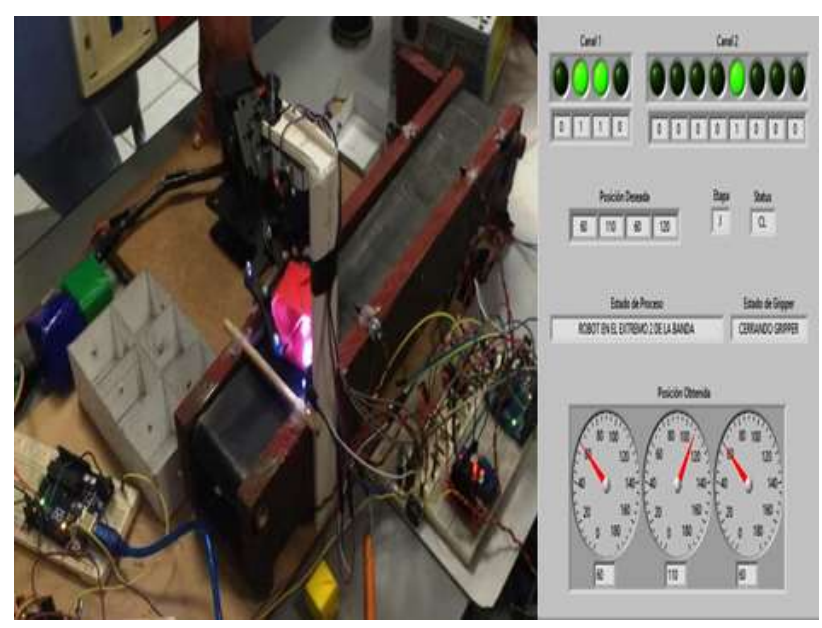

Figura 9 Recuperación de objeto del segundo extremo de la banda

Fuente: Elaboración Propia, 2020

\section{Resultados de clasificación de objetos}

A continuación se presentan los resultados de operación del prototipo implementado, posterior a la ejecución de la tarea de inspección, misma que determina el destino que cada pieza analizada habrá de tener, de acuerdo al color detectado. La figura 10, muestra al robot sobre depósito de las piezas rojas; la figura 11, corresponde al destino dado a las piezas verdes; y finalmente, la figura 12 , representa el confinamiento de las piezas pertenecientes a la tercera clase cuyo color es el azul. Se caracteriza la precisión del movimiento ejecutado por el robot para dar alcance oportuno a la localización de cada almacén en particular. 


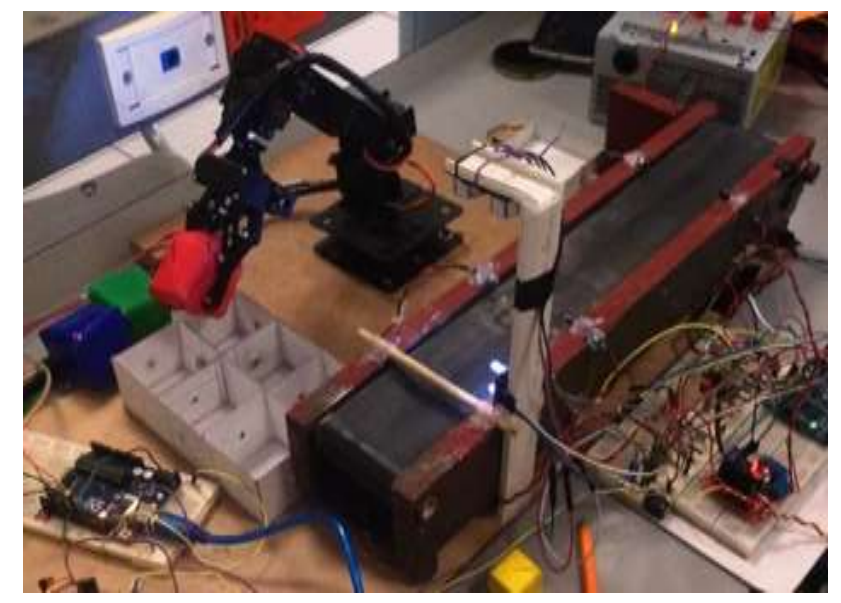

Figura 10 Clasificación de objetos en color rojo Fuente: Elaboración Propia, 2020

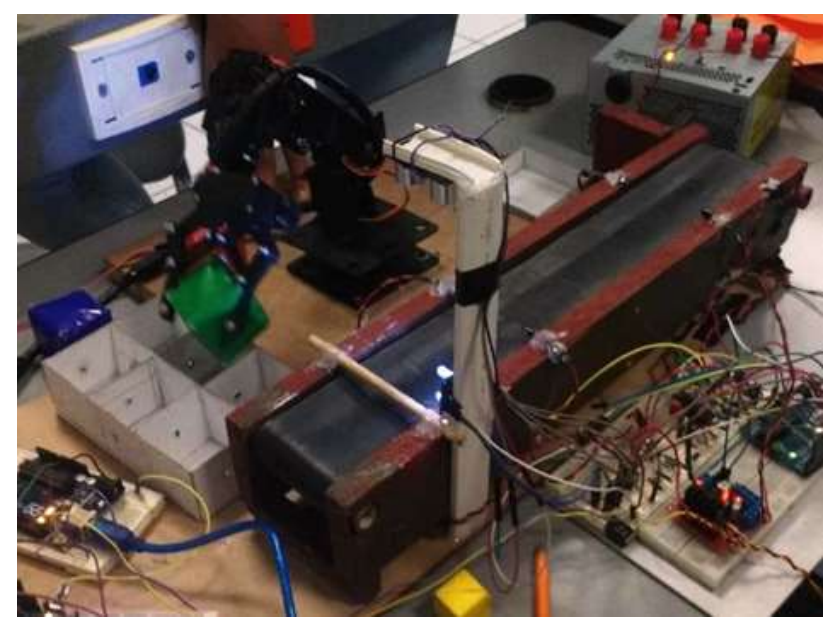

Figura 11 Clasificación de objetos en color verde Fuente: Elaboración Propia, 2020

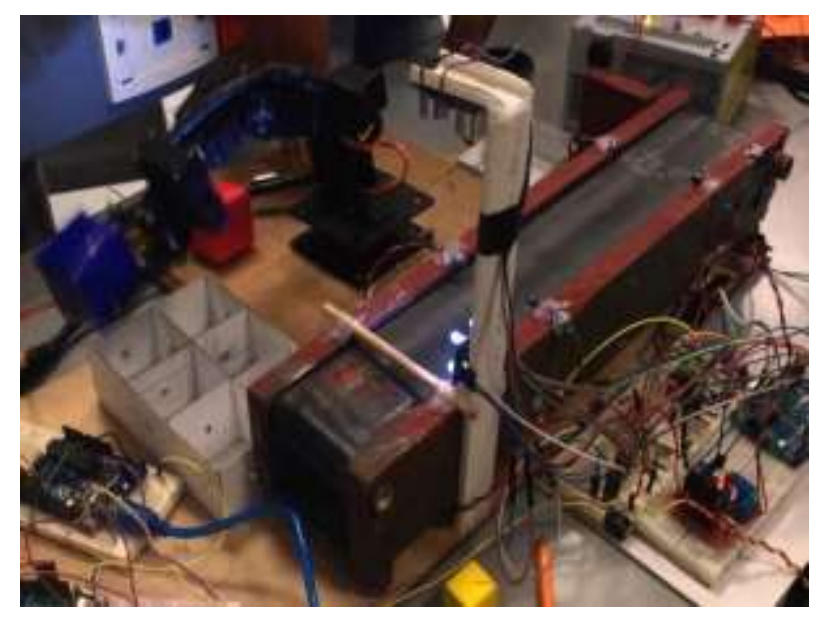

Figura 12 Clasificación de objetos en color azul Fuente: Elaboración Propia, 2020

De forma posterior a la propuesta y el ingreso a la interfaz de las posiciones a alcanzar, por parte del efector final del robot, así como de ajustes mínimos en la calibración del sensor de color para la adecuada lectura e interpretación de los valores correspondientes, se aseguró la efectividad en la secuencia del proceso de clasificación expuesto, para la totalidad de las pruebas desarrolladas.

Cabe mencionar, que la correcta calibración del sensor de color utilizado eliminó toda posibilidad de que la función del sistema de control entrara en confusión, esto al detectar los colores verde o azul en el objeto bajo estudio. La ocurrencia de este fenómeno es habitual al emplear cualquier dispositivo para la detección de color, si las condiciones de iluminación no son cuidadosamente aplicadas; lo cual, se propicia por el hecho de que los espectros electromagnéticos de ambos colores son muy próximos (de 240 a $495 \mathrm{~nm}$ y de 495 a $570 \mathrm{~nm}$, respectivamente).

\section{Conclusiones}

La aplicación del estudio de sistemas de control en eventos discretos al diseño de controladores de proceso actuales, ha permitido la incorporación de metodologías formales a procesos de manufactura, mismas que proveen una adecuada definición de las funciones que cada operación constitutiva deba desempeñar, una vez alcanzadas determinadas condiciones. Tal soporte teórico, lleva a asegurar una secuencia específica de las tareas del sistema productivo en que sea adaptado, para garantizar que su operación total inicie, transcurra y finalice con una baja o nula ocurrencia de errores debidos a la arquitectura de control establecida, como resultado del análisis emprendido.

Entre los grandes beneficios de la adopción de metodologías formales como GRAFCET, descrita en este trabajo, se encuentra la agilización la interacción entre componentes, dispositivos y equipos integrantes, la rápida y oportuna detección de fallas que puedan suscitar en el proceso ejecutado y la preservación en el cumplimiento de las especificaciones propuestas al principio del estudio. De esta manera, la metodología GRAFCET ha probado su efectividad al ser adaptada en infinidad de procesos industriales donde la sincronización entre más de un equipo es una característica primordial. Para la presente aplicación, el empleo de dicha especificación permitió el adecuado establecimiento de una secuencia de operaciones en un proceso de manejo de materiales, aún a nivel prototipo, a partir de la plena identificación de los parámetros y variables implicadas, la propuesta de los sistemas activos a emplear, el conocimiento de sus posibilidades de actuación, y la conformación de un sistema electrónico de gestión del proceso gestionado a través de una interfaz gráfica por computadora.

RODRÍGUEZ-FRANCO, Martín Eduardo, MARTÍNEZ-ROMERO, Adrian, NÁJERA-MONTOYA, Adolfo Ángel y LÓPEZ-ÁLVAREZ, Yadira Fabiola. Sincronización de sistemas activos en procesos de clasificación de objetos por color. Revista de Tecnologías en Procesos Industriales. 2020 
Además, de complementar la acción de la operación descrita, a partir de la adaptación de un sistema de clasificación de objetos por atributo de color; lo cual posibilitó la flexibilidad del proceso intervenido, al disponer de una variedad de destinos por seguir para cada pieza que fuese analizada. Desde una perspectiva funcional, la deducción de un modelo gráfico que concentrara la información de una secuencia previamente definida, trajo consigo la ventaja de disponer de la misma realizando su adecuada traducción a los algoritmos que permitieron la programación de los controladores utilizados.

Tal integración, aunque persigue fines didácticos, resulta ser una viable opción para la aplicación de los conocimientos valorados en asignaturas de corte tecnológico dentro del ámbito de la Ingeniería. Sin embargo, es de resaltar, que en ningún momento, es pretensión del prototipo desarrollado sustituir la labor que se puede realizar en caso de disponer de equipamiento industrial necesario. Más resulta preciso afirmar que esta plataforma puede fungir como un material de apoyo para la consideración de puntos finos de operación y de seguridad, previa al uso de un equipo más robusto, logrando comprender las implicaciones a considerar al momento de poner a interactuar dos o más entidades dentro de un proceso productivo.

\section{Referencias}

Alvarez, M., Burgos, A., Sarachaga, I., Estévez, E., \& Marcos, M. (2012). GEMMA based approach for generating PLCopen Automation projects. IFAC Proceedings Volumes, 230-235.

Björnsson, A., Jonsson, M., \& Johansen, K. (2018). Automated material handling in composite manufacturing using pick-and-place systems - a review. Robotics and ComputerIntegrated Manufacturing, 222-229.

Campos, J. T., Santos, C., de Souza, C., \& da Silva, R. (2013). Design of reconfigurable manufacturing control applied in a material handling production system using Petri nets. IFAC Proceedings Volumes, 499-504.

Corona Ramírez, L. G., Abarca Jiménez, G. S., \& Mares Carreño, J. (2014). Sensores y Actuadores. Aplicaciones con Arduino. México D.F.: Grupo Editorial Patria.
Endsley, E. W., Almeida, E. E., \& Tilbury, D. M. (2006). Modular finite state machines: Development and application to reconfigurable manufacturing cell controller generation. Control Engineering Practice, 1127-1142.

Fitouhi, M.-C., Nourelfath, M., \& Gershwin, S. B. (2017). Performance evaluation of a twomachine line with. Reliability Engineering \& System Safety, 61-72.

Hrúz, B., \& Mrafko, L. (2003). Solution of the manufacturing transportation control using Petri nets. IFAC Proceedings Volumes, 223-228.

Huang, Y., Chiba, R., Arai, T., Ueyama, T., \& Ota, J. (2015). Robust multi-robot coordination in pick-and-place tasks based on partdispatching rules. Robotics and Autonomous Systems, 70-83.

Jamhour, A., \& Garcia, C. (2012). Automation of industrial serial processes based on finite state machines. Procedia Engineering, 186-196.

Ore, F., Hansson, L., \& Magnus, W. (2017). Method for design of human-industrial robot collaboration workstations. Procedia Manufacturing, 11(1, p. 4-12), 4-12.

Pichard, R., Ben Rabah, N., Carre-Menetrier, V., \& Riera, B. (2016). CSP solver for Safe PLC Controller: Application to manufacturing systems. IFAC-PapersOnLine, 402-407.

Qamsane, Y., El Hamlaoui, M., Tajer, A., \& Philippot, A. (2017). A tool support to distributed control synthesis and Grafcet implementation for discrete event manufacturing systems. IFAC-PapersOnLine, 5806-5811.

Qamsane, Y., Tajer, A., \& Philippot, A. (2016). Distributed supervisory control synthesis for discrete manufacturing systems. IFACPapersOnLine, 396-401.

Riera, B., Annebicque, D., Gellot, F., Philippot, A., \& Benlorhfar, R. (2012). Control synthesis based on logical constraints for safe manufacturing systems. IFAC Proceedings Volumes, 194-199. 
Riera, B., Coupat, R., Philippot, A., Annebique, D., \& Gellot, F. (2014). Control design pattern based on safety logical constraints for manufacturing systems: application to a palletizer. IFAC Proceedings Volumes, 388-393.

Schneider, G. F., Peßlera, G. A., \& Terkaj, W. (2019). Knowledge-based conversion of finite state machines in manufacturing automation. Procedia Manufacturing, 189-194.

Schumacher, F., Schröck, S., \& Fay, A. (2013). Transforming hierarchical concepts of GRAFCET into a suitable Petri net formalism. IFAC Proceedings Volumes, 295-300.

Silva, A. L., Ribeiro, R., \& Teixeira, M. (2017). Modeling and control of flexible contextdependent manufacturing systems. Information Sciences, 1-14.

Zaytoon, J. (2010). On the recent advances in Grafcet. Production Planning \& Control, 86100.

Zhang, X., Yang, M., Zhang, J., \& Shi, H. (2012). A study on the extended unique input/output sequence. Information Sciences, 44-58. 